Physical Geography; Cartography; Geographic Information Systems \& Spatial Planing

\title{
COMPARISON BETWEEN PRESTACK DEPTH MIGRATION AND DIRECT DEPTH CONVERSION OF TIME MIGRATED SECTION USING TOMOGRAPHY BASED VELOCITY MODEL
}

DOI: http://dx.doi.org/10.18509/GBP.2016.09 UDC: $550.344 .094 .5:[621.386 .1: 004.6$

Assist. Prof. Dr. Maya Grigorova

University of Mining and Geology "St. Ivan Rilski”, Bulgaria

\begin{abstract}
Depth migration is a key issue for obtaining high resolution data, used for more confident and reliable structural interpretation. Depth processing enhances the image of the subsurface in areas where both the vertical and lateral velocity models are changing. For better migration precise velocity model is needed. Velocity model based on tomography solution has proven itself to create detailed velocity field for determination of rapid velocity changes in complex regions. In this study tomography inversion is used to create depth velocity model for Prestack Depth Migration, as well as for quick depth look using time-to-depth conversion of time migrated section.
\end{abstract}

Keywords: Depth migration, tomography, velocity model, depth conversion

\section{INTRODUCTION}

Depth conversion, ideally, is an iterative process that begins with proper seismic processing in time domain, seismic velocity analysis and study of well data to refine the conversion. In many today's exploration areas, especially where structures are complex and seismic velocity vary abruptly because of faulting, time domain processing can give misleading results and only depth imaging can define the true position and correct geometry of subsurface features [1]. The major problem with this approach is that its implementation requires the construction of a velocity model for the seismic section. It can be both difficult and time consuming to construct an adequate velocity model which tie perfectly during the migration process.

The main idea of this work is to construct a velocity model from time migration velocities as a starting model. Using these velocities then is performed depth migration to obtain an improved seismic gathers for velocity model detailisation using tomography inversion. As an alternative to depth migration, one can instead directly convert a time migrated image to "depth" using the updated by tomography depth velocity filed.

\section{VELOCITY MODEL BUILDING TECHNIQUES}

In many cases time migrations are inadequate for accurate geological interpretation of subsurface structures. In areas of rapid lateral and vertical velocity changes, energy is dispersed in such a way that time migrated data cannot provide desired resolution of the data. Depth migration can handle lateral velocity variations and is prone to bring significant improvement of seismic data quality (both resolution and continuity) and proper spatial structural positioning. But for these purposes it requires an accurate interval velocity model construction, which is in practice both challenging and time- 
consuming [2]. An iterative process of tomographic updates is often employed, where a good initial interval velocity model is crucial for achieving reliable and interpretative results. It is thus of great interest to convert the time-migration velocity to the depth domain in order to unravel inherent distortions in time-domain images and to provide a reasonable starting model for building depth-imaging velocities [3].

In this study RMS velocities derived by migration velocity analysis (MVA) are converted to interval velocities in depth and are used as a starting point for tomography update and forward PSDM and depth conversion process.

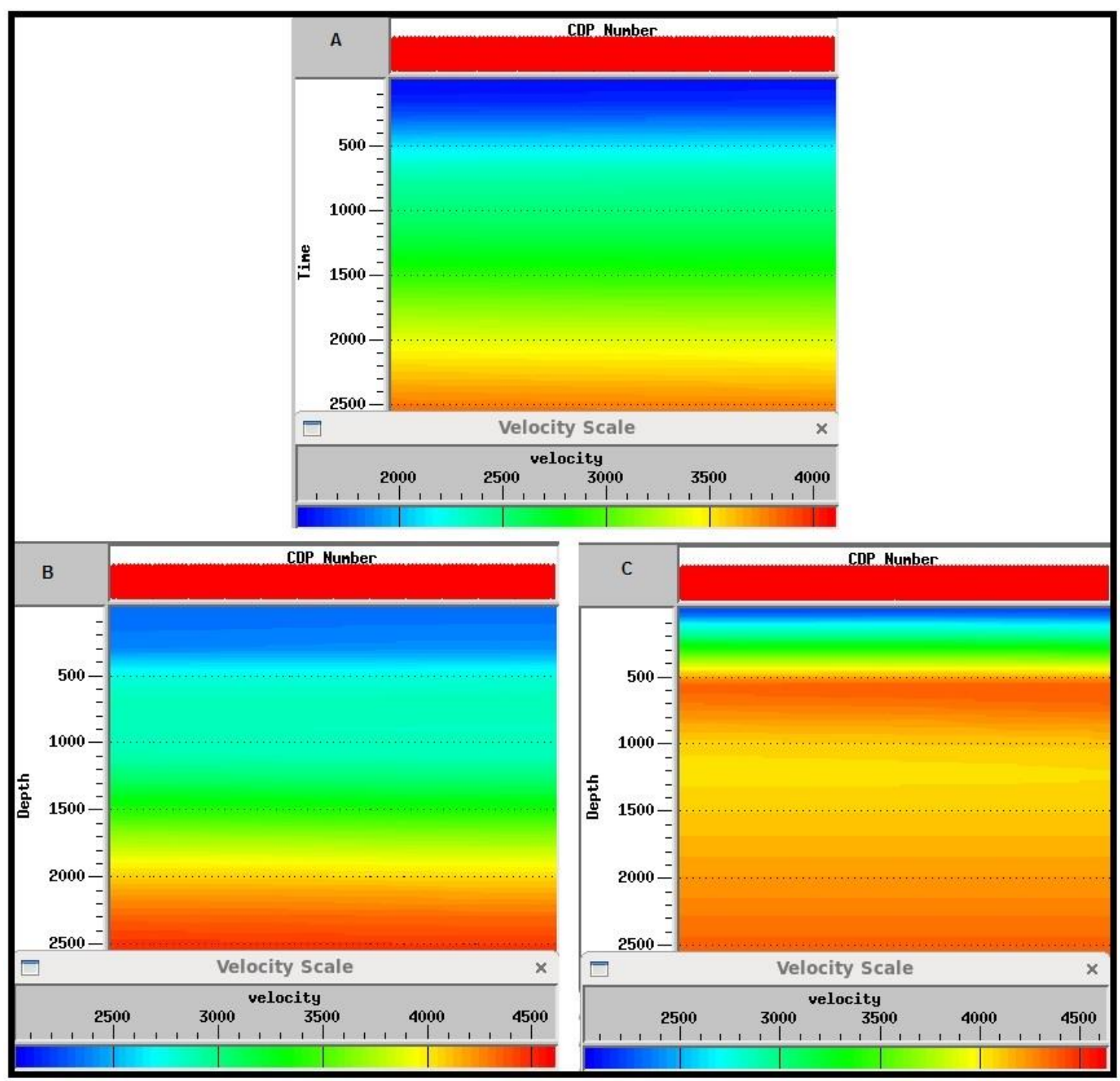

Figure 1. Velocity models: A - initial velocity field derived by MVA; B - depth converted interval velocity; $\mathrm{C}$ - velocity model obtain by tomography inversion

Since most of today's seismic projects are run through PSDM, an elegant way to derive the velocity volume for time-to-depth conversion is to use the same tool used for PSDM velocity model building, the tomography inversion [4]. 


\section{DEPTH IMAGING OR DEPTHING}

Although velocity model obtain by seismic data (RMS velocity) will always have more error and uncertainty than well velocities, for time-to-depth conversion purposes one can convert them either to average or to interval velocities and then benefit from their added spatial coverage.

Depthing can be done via a wide range of existing methods. In general they can be separated into two categories:

- direct time-to-depth conversion using well velocities;

- velocity modeling for time-depth conversion.

Direct time-to-depth conversion usually ignores the spatial extends of the velocity, and operates at known depth points only (i.e. at wells) by forcing an exact or minimal error match between actual and predicted depths.

Velocity modeling for time-depth conversion involves building velocity model using all available velocity data.

The choice of a depthing method depends on data availability and quality, depthing objectives, and time and cost constraints on the depthing process. Direct methods are fast and accurate at the well location, but often fail in spatial distribution. Some forms of velocity modeling can also be fast and exact, whereas other forms require significant data resources, modeling expertise, and attention to details.

In this study velocity modeling process starts from RMS stacking velocities obtain by MVA. This velocity field is then used for tomography updates and detailisation.

On figure 2 is shown comparison between time-to-depth conversion of seismic section using tomography based velocity model and time-to-depth conversion of the same section using direct converted to depth RMS velocities.

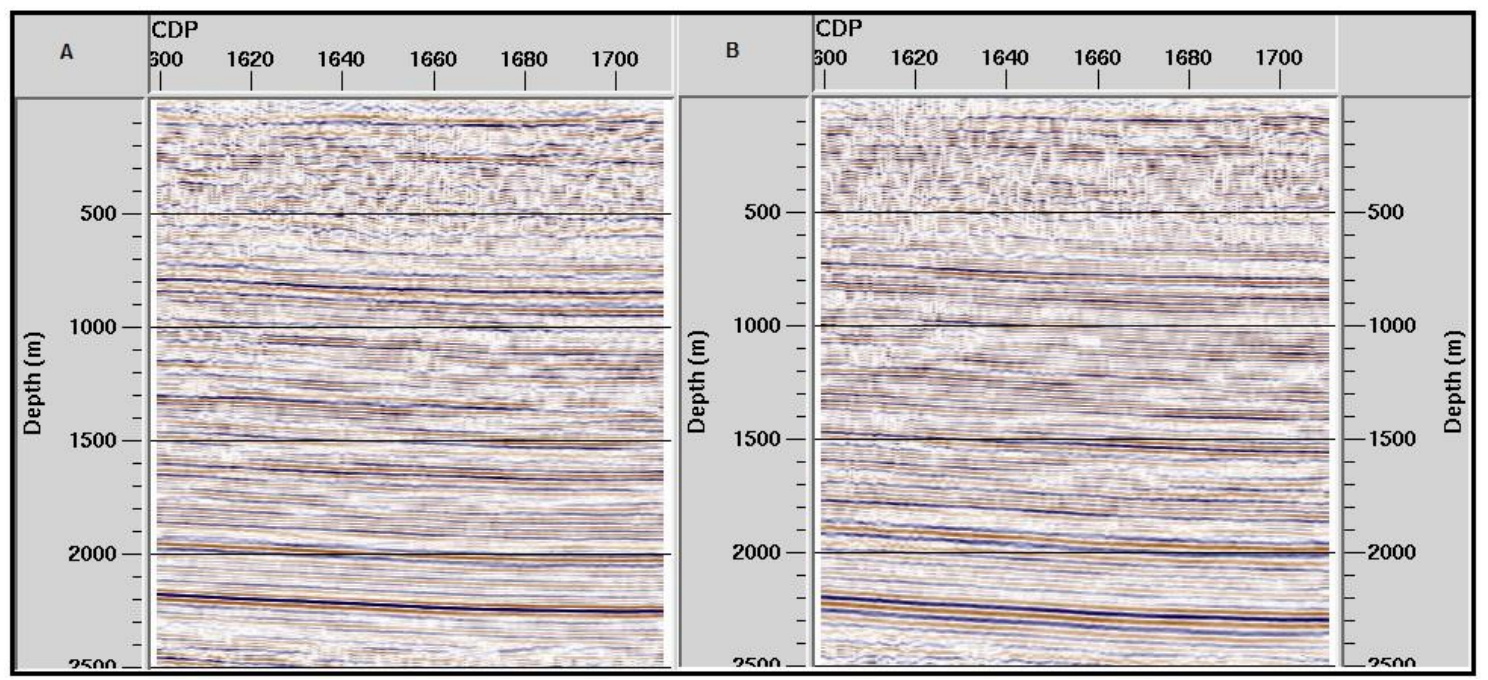

Figure 2. Comparison between: A - Time-to-depth conversion using tomography based velocity model;

B - Time-to-depth conversion using direct converted to depth RMS velocities

The comparison on figure 2 shows that the new derived by tomography inversion velocity model significantly improve image quality in deep part of the section.

No depth conversion or depthing can correct for improper lateral positioning of events, because depth conversion is a vertical process only. Depth migration is currently the ultimate tool for lateral imaging, but it does not calibrate the seismic to true depth, because 
it does not use true vertical propagation velocities. This is not an error - imaging is a separate issue from true depth calibration [5].

Depth imaging sometimes is long and laboring process which in general would be as good as the time processing that preceded it.

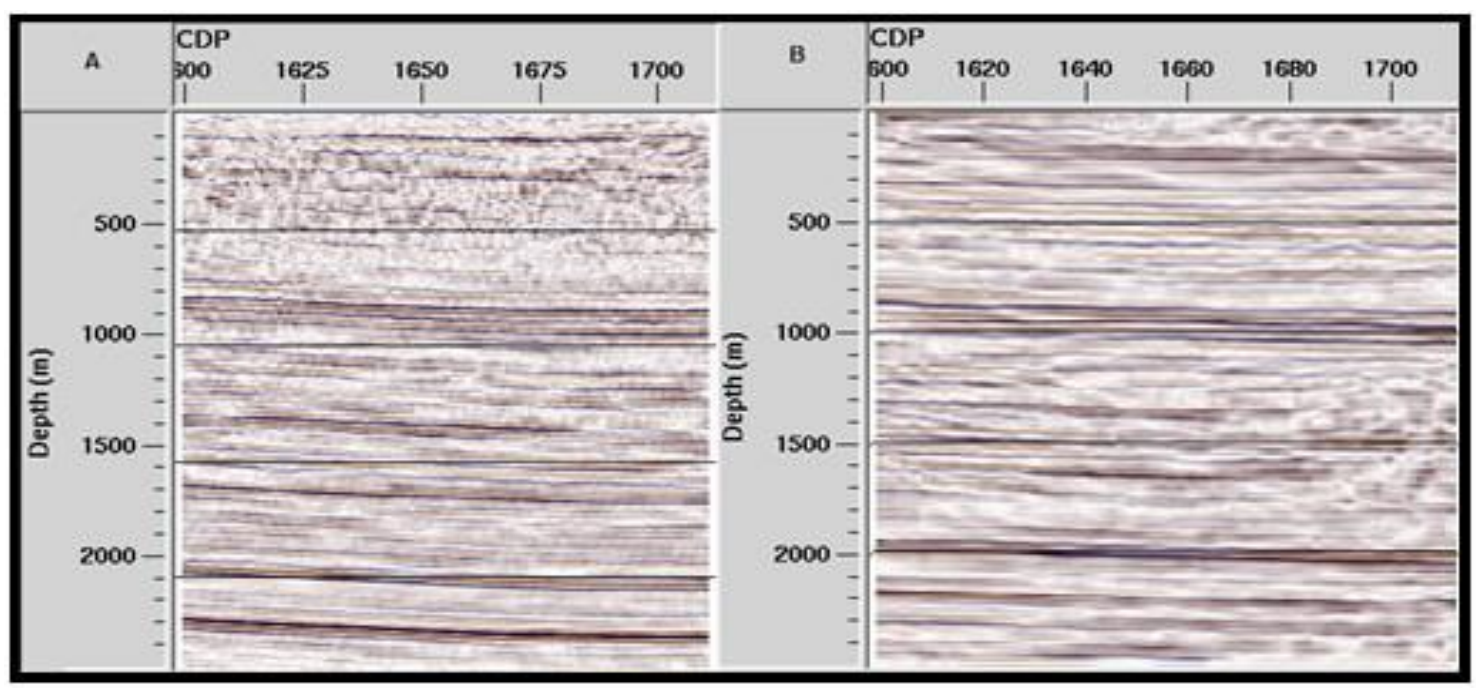

Figure 3. Comparison between: A - Time-to-depth conversion with velocity field obtain by tomography inversion and B - PSDM with velocity field obtain by tomography inversion

On the comparison shown on figure 3 can be noticed that in the shallow part of the section there is better definition of reflection boundaries on the PSDM section obtain with velocity field derived by tomography than the time-to-depth converted section obtain with the same velocity field. However in mild lateral velocity variations depth converted with tomography based velocity model seismic section can be representative for quick look in depth.

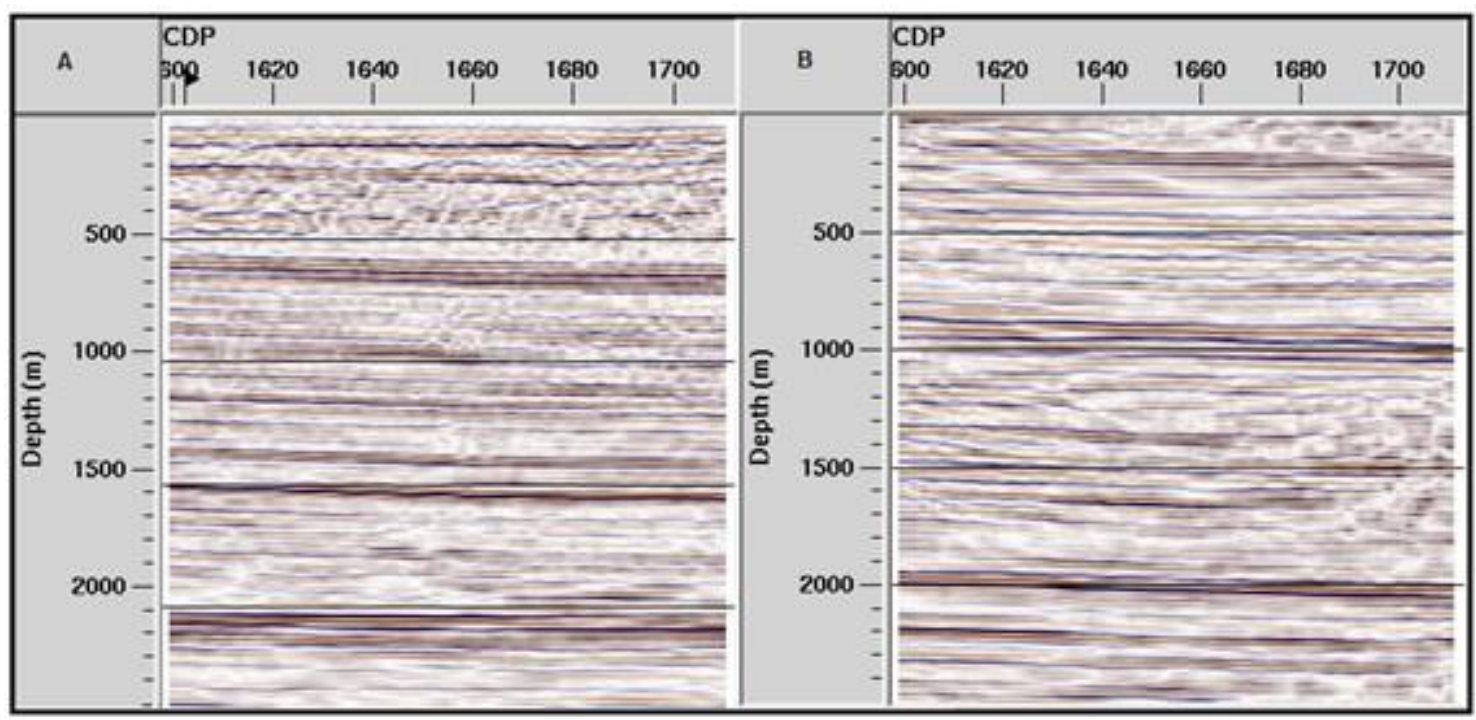

Figure 4. A - Pre-stack time migration; B - Pre-stack depth migration

Compared to the pre-stack time migration, the final pre-stack depth migrated section showed a significantly enhanced definition of the main structures, as well as improved continuity of the reflectors. The final velocity model, constrained by seismic data, also 
enabled a more accurate estimation of the structures in depth. This information enabled user to make a more accurate economic assessment of the subsurface environment.

\section{CONCLUSION}

In every seismic investigation seismic data is acquired in time - the time taken for the sound to travel from the source to reflectors and to return to receivers throw the subsurface. However, geological structures and layers appear in depth. Therefore, conversion from time to depth is needed for a clear picture of the prospect.

Nevertheless, there is no single methodology that is optimal for all cases since the available seismic and geologic data varies in quantity and quality within each project. To design an effective approach to depth conversion, the first thing is to understand the nature of velocity field. Next, appropriate depth-conversion method is needed to tailor the specific geological and geophysical constraints.

Depth imaging, once too costly and difficult for commercial use, is now the preferred seismic imaging tool for today's most seismic projects. In structurally complex areas, traditional time domain processing gives misleading results and only depth imaging is able to reveal the true location and shape of subsurface features.

\section{REFERENCES}

[1] Albertin, U., Kapoor, J., Randall, R., Smith, M., Brown, G., Soufleris, Ch., Whitfield, P., Dewey, F., Farnsworth, J., Grubitz, G., Kemme, M., The time for depth imaging, Oilfield review, 2002

[2] Grigorova, M. Time - to - Depth migration using tomography based velocity model, 7th Congress of Balkan Geophysical Society, Albania, 2013, No 18499.

[3] Li, S., Fomel, S., A robust approach to time-to-depth conversion and interval velocity estimation from time migration in the presence of lateral velocity variations a Published in Geophysical Prospecting, 63, no. 2, 315-337, 2015

[4] Mancini, F., High resolution time-to-depth conversion using 3D Grid Tomography. ASEG Extended Abstracts , 1-4, 2013

[5] Edward L. Etris, Nick J. Crabtree and Jan Dewar, True Depth Conversion: More Than a Pretty Picture, CSEG Recorder, vol. 26, no. 09, 2002 